Proceedings

\title{
Fluorescent Chiral Nanostructures Based on Poly(diphenylacetylene)s ${ }^{\dagger}$
}

\author{
Juan José Tarrío ${ }^{1}$, Emilio Quiñoá ${ }^{2}$ and Félix Freire ${ }^{2, *}$ \\ 1 Universidade de Santiago de Compostela; juanjose.tarrio@usc.es \\ 2 Universidade de Santiago de Compostela; emilio.quinoa@usc.es \\ * Correspondence: felix.freire@usc.es; Tel.: +34-881815763 \\ + Presented at the 24th International Electronic Conference on Synthetic Organic Chemistry, 15 November- \\ 15 December 2020; Available online: https://ecsoc-24.sciforum.net/.
}

Received: date;

\begin{abstract}
Metals are one of the most employed external stimuli for modifying the structure of helical polymers. In poly(phenylacetylene)s it's known that can act as crosslinking agent among polymer chains, inverse the helical sense or enhance the initial helicity. Finally, nanostructures like nanospheres, nanorods, etc, can be obtained. In order to find new structures and new functions in helical polymers, this work addressed the synthesis of a novel family of materials denominated poly(diphenylacetylene)s. These polymers are a special class of conjugated polymers that present the abilities of fluorescence emission and a high thermal stability. In this work, we analyze the chiroptical properties of the synthetized polymers and how the addition of different metals can modify them. Also, fluorescent chiral nanostructures are obtained.
\end{abstract}

Keywords: helical polymers; fluorescent; chiral; nanostructures; poly(diphenylacetylene)s

\section{Introduction}

During the last decades many helical polymers have been studied due to the similar structure with some biomolecules such as the DNA, proteins or sugars. It is proven the direct relationship between the structure/function of these biomolecules, for this, scientists have developed helical polymers such as poly(phenylacetylene)s, poly(isocyante)s or poly(isocyanide)s among others [1].

Recently, a novel family of helical polymers was added to this group denominated poly(diphenylacetylene)s (PDPAs). These polymers are a special class of conjugated polymers that can present several properties including chirality, fluorescence emission, thermal stability or liquid crystallinity [2].

Basing on previous knowledge using metals with poly(phenylacetylene)s for modifying the properties of these materials [3], we used them to form chiral nanospheres with the poly(diphenylacetylene)s.

\section{Results and Discussion}

After the polymerization of the monomer M-1 (Scheme 1) following the conditions described on literature [4], we made a post-polymerization coupling with an alanine methyl ester derivative obtaining the final polymer P-2 (Figure 1). 


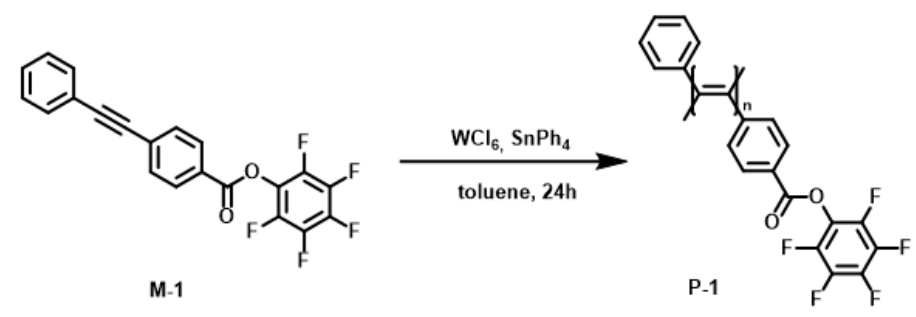

Scheme 1. Text.
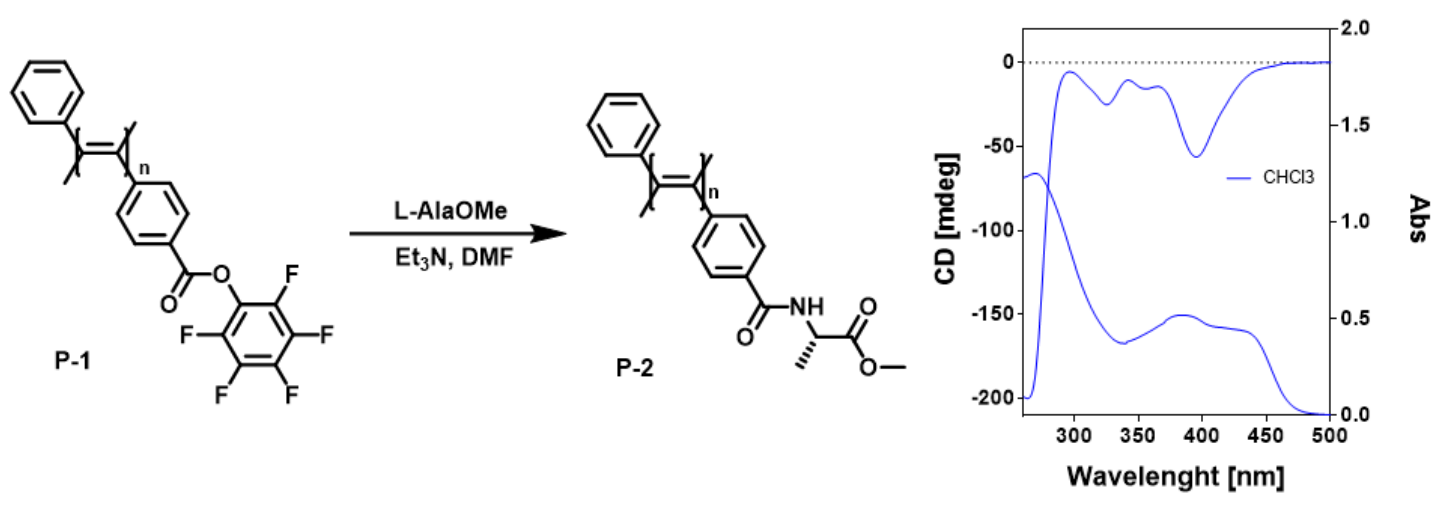

Figure 1. Text.

After a thermal annealing in $\mathrm{CHCl}_{3}$ at $80{ }^{\circ} \mathrm{C}, \mathbf{P}-2$ presents a helical structure that we confirmed by $\mathrm{CD}$ technique (Figure 1).

Preparing several solutions of P-2 $(0.5 \mathrm{mg} / \mathrm{mL})$ and adding different amounts of some metals we could observe the perfect formation of nanospheres by DLS (Figure 2) that have an intrinsic chirality due to the helical structure of the polymer. Also, due to the ability of emission, we could measure the fluorescence of the hybrid material (Figure 2).
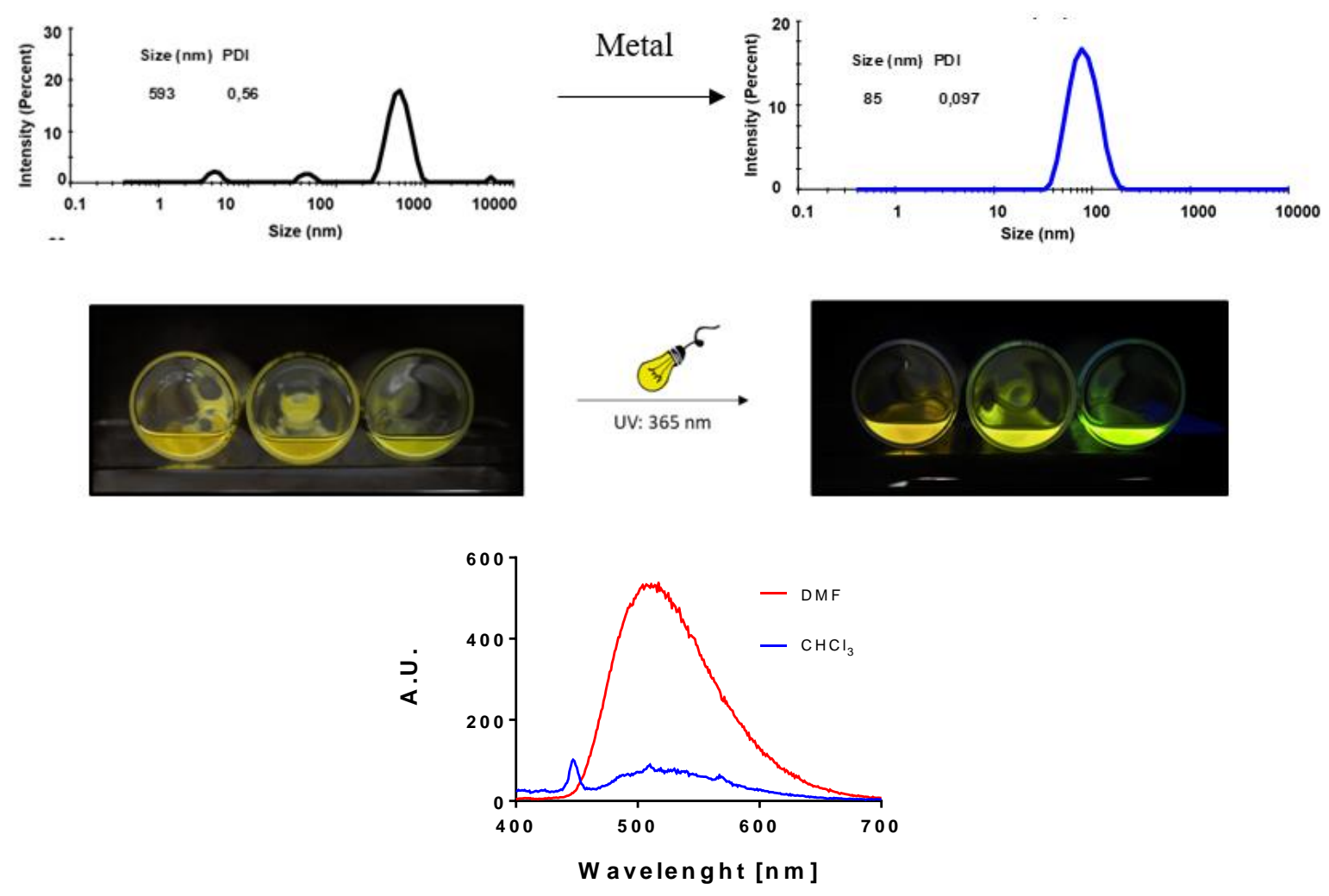

Figure 2. Text. 


\section{Conclusions}

In this study, we have achieved the formation of fluorescent chiral nanospheres applying a previous knowledge using metals as cross-linking agents. Further studies will permit us to see if they are CPL (circularly polarized luminescence) active and if we can control and modulate the size and sense of the particles.

Acknowledgments: We thank MINECO (CTQ2015-70519-P), Xunta de Galicia (ED431C 2018/30, Centro singular de investigación de Galicia accreditation 2019-2022, ED431G 2019/03) and the European Union (European Regional Development Fund-ERDF) for financial support and Ministerio de Educación y Formación Profesional for an FPU predoctoral fellowship.

\section{References}

1. Yashima, E.; Ousaka, N.; Taura, D.; Shimomura, K.; Ikai, T.; Maeda, K. Supramolecular Helical Systems: Helical Assemblies of Small Molecules, Foldamers, and Polymers with Chiral Amplification and Their Functions. Chem. Rev. 2016, 116, 13752-13990.

2. Jin, Y.J.; Kwak, G. Properties, Functions, Chemical Transformation, Nano-, and Hybrid Materials of Poly(diphenylacetylene)s toward Sensor and Actuator Applications. Polym. Rev. 2017, 57, 175-199.

3. Arias, S.; Núñez-Martínez, M.; Quiñoá, E.; Riguera, R.; Freire, F. A general route to chiral nanostructures from helical polymers: P/M switch via dynamic metal coordination. Polym. Chem. 2017, 8, 3740-3745.

4. Zhang, X.A.; Qin, A.; Tong, L.; Zhao, H.; Zhao, Q.; Sun, J.Z.; Tang, B.Z. Synthesis of Functional Disubstituted Polyacetylenes Bearing Highly Polar Functionalities via Activated Ester Strategy. ACS Macro Lett. 2012, 1, 75-79.

Publisher's Note: MDPI stays neutral with regard to jurisdictional claims in published maps and institutional affiliations.

(C) 2020 by the authors. Submitted for possible open access publication under the terms and conditions of the Creative Commons Attribution (CC BY) license (http://creativecommons.org/licenses/by/4.0/). 